\title{
Hidden Subsurface Reconstruction and Its Atomic Origins in Layered Oxide Cathodes
}

Linze $\mathrm{Li}^{1}$, Ethan Self ${ }^{2}$, Devendrasinh Darbar ${ }^{2}$, Lianfeng Zou ${ }^{1}$, Indranil Bhattacharya ${ }^{3}$, Donghai Wang ${ }^{4}$, Jagjit Nanda $^{2}$ and Chongmin Wang ${ }^{1}$

${ }^{1}$ Pacific Northwest National Laboratory, Richland, Washington, United States, ${ }^{2}$ Oak Ridge National Laboratory, Oak Ridge, Tennessee, United States, ${ }^{3}$ Tennessee Technological University, Cookeville, Tennessee, United States, ${ }^{4}$ The Pennsylvania State University, University Park, Pennsylvania, United States

Structural transformations near surfaces of solid-state materials underpin functional mechanisms of a broad range of applications including catalysis, memory, and energy storage. It has been a long-standing notion that the outmost free surfaces, accompanied by broken translational symmetry and altered atomic configurations, are usually the birthplace for structural transformations. Here, in a layered oxide cathode for $\mathrm{Li}$-ion batteries [1], $\mathrm{LiNi}_{0.495} \mathrm{Mn}_{0.495} \mathrm{Mo}_{0.01} \mathrm{O}_{2}$ (LNMMO), we for the first time observe the incipient state of the well documented layered-to-spinel-like structural transformation, which is surprisingly initiated from subsurface layer, rather than the very surface.

Atomic-scale STEM HAADF, ABF, inverted ABF images, and corresponding intensity profiles along the atomic planes as marked with " 1 " and "2" lines in the HAADF and inverted-ABF images of a local nanoregion below the surface of a LNMMO particle are shown in Fig. 1a-d, respectively. From these images and intensity profiles, an arrangement of 2 monolayers of rocksalt structures at the outmost surface, followed by 3 monolayers of a transitional spinel-like structure, and then 5 monolayers of layered structures with strong cation mixing, and lastly a few monolayers of incipient-spinel structures, is deduced. Further inside, a gradual transition from the incipient-spinel structures to the layered structures with less cation mixing is observed. To explore the origin of such atomic rearrangements, EELS spectra of O $\mathrm{K}$ edges and $\mathrm{Mn}$ and $\mathrm{Ni} \mathrm{L}$-edges are collected at 12 different locations (marked with numbers in Fig. 1a) below the surface (Fig. 2a-c). The extracted composition ratios (i.e., $\mathrm{Mn} / \mathrm{O}$ and Ni/O ratios) and $L_{3} / L_{2}$ ratios of Mn are plotted in Fig. 2d and e, respectively. Corresponding low-loss EELS spectra of Mn $M$ edges and Li $K$-edges are shown in Fig. 2f, where the spectra are normalized with the Mn $M$-edges. These EELS results clearly show some features that can be correlated to the structural transitions from the inner region to the outmost surface. First, the intensity of the $\mathrm{O}$ prepeaks indicated by the arrow in Fig. 2a is gradually suppressed from the inner region to the surface, suggesting an increased accumulation of $\mathrm{O}$ vacancies. Second, the intensity of the Li $K$-edges is also gradually reduced from the inner region to the surface, indicating the formation of a local gradient distribution of $\mathrm{Li}$, where the inner region is Li-retained and the surface is Li-depleted. Third, non-uniform distributions for both $\mathrm{Mn}$ and $\mathrm{Ni}$ are observed from the inner region to the surface (Fig. 2d), which can also be correlated to the distribution of different structures at the surface and subsurface.

In conclusion, by coupling atomic level STEM imaging with EELS, we discover that the reconstructed structures at the subsurface of layered cathodes are associated with selective atomic species partition and consequent nanoscale non-uniform composition gradient distribution at the subsurface. Our findings provide fundamental insights on atomic-scale mechanisms of structural transformation in layered cathodes [2]. 

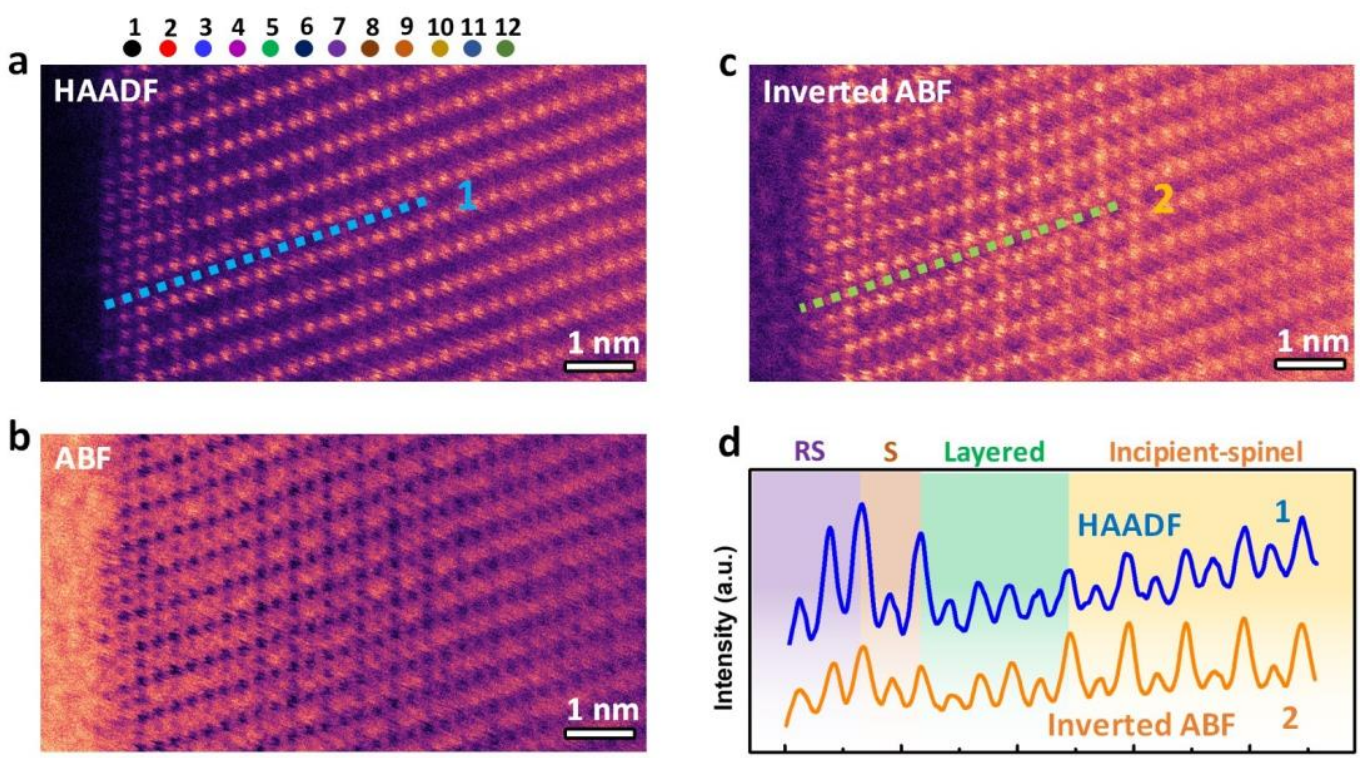

V $\mid$ RS S: S: Layered Incipient-spinel! Layered

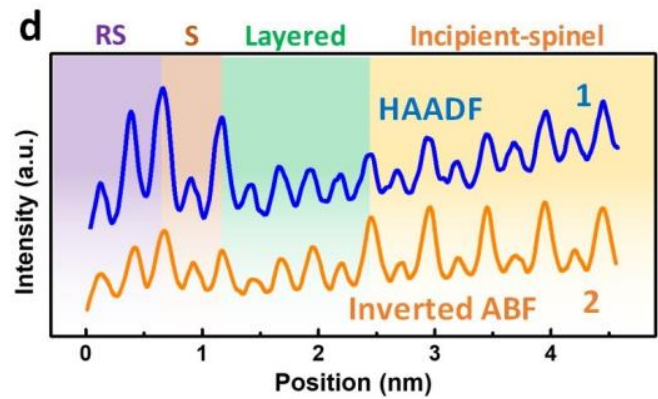

Figure 1. (a-c) STEM HAADF (a), ABF (b), and inverted-ABF (c) images of the same LNMO surface. In (b), "V", "RS", and "S" are short for vacuum, rocksalt, and spinel, respectively. (d) Image intensity profiles along the Li layers as marked with " 1 " and " 2 " lines (a) and (c). The profiles are averaged across multiple Li layers.

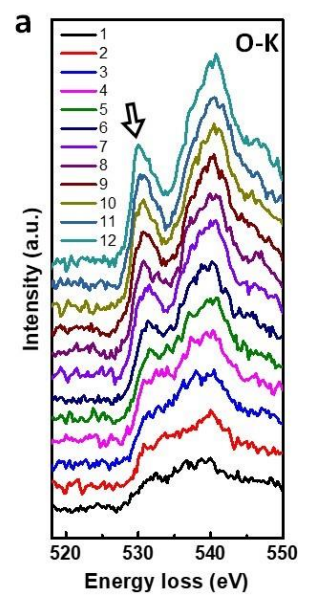

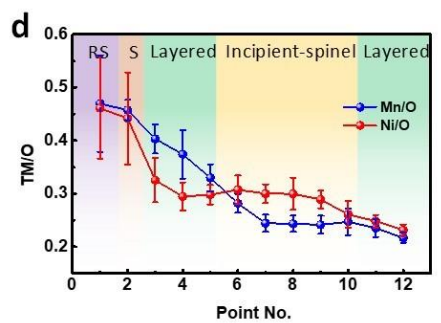
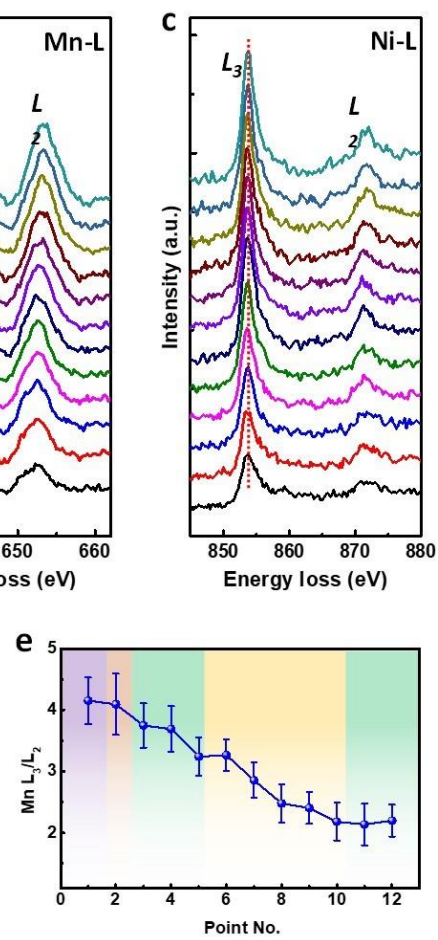

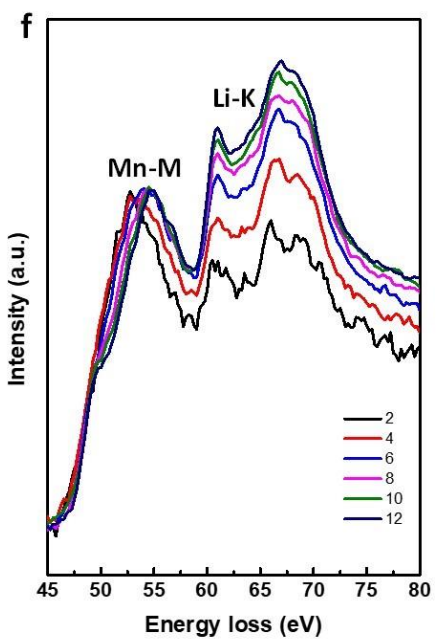

Figure 2. (a-c) EELS spectra of O K-edges and Mn and Ni L-edges collected at 12 different locations as marked in Fig. 1(a). The black arrow in (a) marks the O prepeaks. The dashed lines in (b) and (c) mark the L3 peak positions. (d,e) Extracted composition ratios (i.e., $\mathrm{Mn} / \mathrm{O}$ and Ni/O ratios) (d) and L3/L2 ratios of Mn (e) at these 12 different locations. In (d), "RS" and "S" are short for rocksalt and spinel, respectively. 
(f) Low-loss EELS spectra of Mn M-edges and Li K-edges collected at 6 different locations (even numbers marked in Fig. 1(a)), where the spectra are normalized with the Mn M-edges.

\section{References}

[1] Li, L. Z. et al, Acs Energy Letters 4, 2540-2546, (2019).

[2] This work is supported by the Department of Energy under Award Number DE-EE0008447. 